\title{
INFRARED SYNCHROTRON RADIATION INSTRUMENTATION AND APPLICATIONS
}

\author{
Gwyn P. Williams \\ National Synchrotron Light Source \\ Brookhaven National Laboratory \\ Upton, New York 11973, USA
}

BNL --46592

DE92 000474

\begin{abstract}
Infrared synchrotron radiation (IRSR) in the wavelength range from $1 \mu \mathrm{m}$ to $-1 \mathrm{~cm}$ has now been used quite extensively both in Japan at UVSOR, Okasaki and in the United States at the NSLS, Brookhaven. Elsewhere around the world, several new beamlines are under construction or planned - including those at LUND (Sweden), Daresbury (UK), SuperACO (France), ADONE (Italy), and the ALS (USA). The use of IRSR poses many new challenges, none the least of which is the fact that the radiation covers four decades of energy and is also very divergent and subject to diffraction. However the advantages are particularly significant due to the 1000 fold increase in brightness available over conventional sources in a region where detectors become a limiting factor. In addition, IRSR is also highly spatially coherent allowing the possibility of a new class of interferometers based on wavefront division. We will discuss this and other instrumentation issues as they critically relate to experiments. The applications discussed will be in the areas of surface vibrational spectroscopy, both in ultra-high vacuum and in electro-chemical cells, and in areas which use the pulsed nature of the source both for fast response studies and for pump-probe studies.

\section{DISCLAIMER}

\footnotetext{
This report was prepared as an account of work sponsored by an agency of the United States Government. Neither the United States Government nor any agency thereof, nor any of their employees, makes any warranty, express or implied, or assumes any legal liability or responsibility for the accuracy, completeness, or usefulness of any information, apparatus, product, or pross disclosed or represents that its use would not infringe privately owned rights. Reference herein to any specific commercial product, process, or service by trade name, trademark, manufacturer, or otherwise does not necessarily constitute or imply its endorsement, recommendation. or favoring by the United States Government or any agency thereof. The views and opinions of authors expressed herein do not necessarily state or reflect those of the United States Government or any agency thereof
} 


\section{Introduction}

We begin by discussing novel instrumentation methods which we have developed in the infrared, but which are equally applicable to the VUV and xray spectral regions. We will show how they enable higher resolution data to be obtained with very high signal to noise and will then show examples of experiments which take advantage of these ideas.

Two infrared beamlines have been operating full scientific programs on storage rings for several years, these being the one at UVSOR, Okasaki, Japan ${ }^{1}$, and the one at the NSLS, Brookhaven National Lab, USA ${ }^{2}$. The first spectrum ever published ${ }^{3}$ with synchrotron radiation, however, was from BESSY, Berlin, Germany. In parallel with the research effort at the NSLS we have continued to study and develop new instrumentation for our beamline. Our goals are to improve the range, resolution and above all signal-to-noise of our measuring equipment. We will address these three issues individually in this paper in each case explaining how they carry over to the VUV and xray spectral regions. Spatial and temporal coherence play a major role in these developments and we therefore briefly explain them. Finally, we discuss the emerging new instrumentation being developed for time dependent studies, including a pump-probe system using a sychronized Nd- YAG laser as the pump.

\section{Range: Spatial Coherence and the Wavefront Dividing Interferometer}

In the infrared spectral region it is possible to use Michelson and other interferometers and these have been the only instruments used recently with synchrotron radiation. They have the "multiplex" advantage in which all wavelengths are sampled for all of the measuring time. Provided source noise dominates, this advantage considerably reduces the measuring time.

A Michelson interferometer, however, requires a "beam-splitter" to produce the two beams. This beamsplitter can divide either the amplitude of the incoming beam, for 
example using a partially reflecting surface, see Fig. 1a, or physically divide the wavefront using a lamellar device such as shown in Fig. 1b. The main problem with amplitude dividing beamsplitters is that the spectral range is limited by the substrate, and often several exchanges of beamsplitters have to be made to cover the desired range. Wavefront dividing beamsplitters also present problems, since the lamellar spacings have to be small enough so that "lamellar pairs" are coherently illuminated and can thus produce an interference pattern. This requirement leads to mechanical problems for short wavelength operation, and hence instruments of this type are normally used only at very long (sub-mm) wavelengths.

However as the following discussion will show, these difficulties are overcome when sy'nchrotron radiation is used. First we discuss what we mean by "coherently iil uminated". A source is said to be spatially coherent across its lateral dimensions if an interference pattern is visible when it illuminates, for example, two slits whose transnitted beams recombine on a screen as shown in Fig. 2a. This condition implies that there is less than $2 \pi$ of phase error across the wavefront. Phase errors result in a loss of visibility of the interference fringes. An alternative way of describing spatial coherence is shown in Fig. 2b. This is a simplification of the general treatment by Born and Wolf ${ }^{4}$ for a screen illuminated by an extended incoherent quasimonochromatic source. In Fig. $2 b$. an observation point, $\mathrm{P}$, is illuminated by light from two points, $S_{1}$, and $S_{2}$, a distance $s$ apart on the source. The phase error resulting from the different optical paths from different points on the source will be small over an area of diameter $2 \mathrm{D}$ given ${ }^{4}$ by:

$$
2 D=\frac{0.16 \lambda R}{s}
$$

Now since the half-angle $\alpha=D / R$, the condition for spatial coherence when $\alpha$ is small can also be written in the form:

$$
s . \alpha \ll \lambda
$$

where $\lambda$ is the wavelength of the light. $s . \alpha$ can be seen in Fig. $2 b$ to be the actual physical path difference between two extreme rays. Although we have defined spatial 
coherence in this way we note that spatial coherence does not imply any phase relationships between emitting portions across the source, only that they are indistinguishable when viewed at a distant screen under the conditions of Eq. 1. Synchrotron radiation has the property that $\alpha$, the half-angle into which the radiation is emitted, is a function of wavelength and varies in such a way as to help maintain spatial coherence, i.e. as $\lambda$ gets shorter, $\alpha$ gets smaller. At $\lambda=1 \mathrm{~cm}$ for example, $\alpha=0.05$ radians while at $\lambda=1 \mu m, \alpha=0.01$ radians. Thus if we assume a height of $200 \mu m$ for the synchrotron source, we see that we satisfy Eq.(1) over the entire range $10 \mu \mathrm{m}$ to $1 \mathrm{~cm}$.

It follows that one can build an instrument such as the one shown schematically in Fig. 3, in which a split mirror is coherently illuminated by the synchrotron, the wave front is divided, and an interferogram obtained by translating one of the mirrors normal to its surface relative to the other. Such an instrument - at normal incidence - is capable of scanning a very wide spectral range, say from $30 \mathrm{~nm}$ to $1 \mathrm{~mm}$ provided the source is spatially coherent. In the VUV/soft xray region undulator sources are ideal in this regard and for wavelengths below 30nm, schemes using grazing incidence optics are reasonably easy to conceive. In addition, by using an optical arrangement in which one of the two beams passes through the sample, one can obtain information about both amplitude and phase changes and hence learn about both real and imaginary part of the dielectric function. We have built such an instrument at the NSLS ${ }^{5}$ and in Figs. 4 and 5 show an inteferogram and its transform. The spectral range was restricted by filters and both beams passed through the sample. These data are identical to those taken with our Nicolet 20F Michelson interferometer.

\section{Resolution: Temporal Coherence}

The relative bandpass $\Delta \lambda / \lambda$ for a wave train of length $L$ is given by $\lambda / L$. It is straight forward to see from Fig. 1 that in an interferometer, $L$ is given by the maximum path difference. Thus at a wavelength of $100 \mu \mathrm{m}$ and a path difference of $10 \mathrm{~mm}$, the resolution $\lambda / \Delta \lambda=.01 / 100 \times 10^{-6}=10^{2}$. Since synchrotron radiation can be relatively well collimated in the infrared, it is possible to use path differences of $1-10$ meters, give $\lambda / \Delta \lambda$ 
values of $10^{4}-10^{5}$. Dieter Moller has built such an instrument for use at the NSLS and it is shown in Fig. 6. The central set of mirrors translates, lengthening the path of the beam on one side while shortening the beam on the other side. The beam-splitting can be done either by amplitude or wavefront division. The first interferogram from this instrument has been obtained recently.

Again we note that for the VUV/soft xray region at a wavelength of $100 \mathrm{~nm}$, a maximum path difference of $10 \mathrm{~mm}$ corresponds to a resolution of $10^{5}$. At $10 \mathrm{~nm}$ a path difference of only $1 \mathrm{~mm}$ gives a $10^{5}$ resolving power!

\section{Signal to Noise}

We have used a novel method to reduce noise in our spectra. One of the dominant contributions to noise in spectra taken with synchrotron radiation is due to beam movement which produces intensity fluctuations. In principle this can be addressed by time averaging at each point in the spectrum. Noting, however, that this often fails because beam movement is not always random about a standard orbit, we have adopted another, opposite philosophy to the above - namely, we take spectra very rapidly, typically scanning the entire range in a few 100 msecs. The source fluctuations which are usually $<100 \mathrm{~Hz}$ in frequency then appear as baseline shifts and are easily corrected for. This method was first suggested by Madden and Strong ${ }^{6}$ in 1954 and has the advantage that no chopper is required so that twice the flux reaches the detector.

With reference to Fig. 1, if we assume that the moving mirror scans at a velocity of $5 \mathrm{~mm}$ per second, the path difference will change by $10 \mathrm{~mm}$ per second. Thus a $100 \mu \mathrm{m}$ wavelength will be modulated and appear as a $100 \mathrm{~Hz}$ signal. Each input wavelength gives a corresponding characteristic frequency and by filtering and frequency analyzing, the spectrum is readily obtained. This principle could easily be exploited for EXAFS measurements, the main requirement being a rapidly tunable monochromator. 
The success of this method is illustrated in Fig. 6, in which we show isotope shifts of vibrational modes for $\mathrm{CO}$ on a $\mathrm{Cu}(100)$ surface ${ }^{7}$. The spectra shown are differences between the reflectivity of a half monolayer $\mathrm{CO}$ covered surface and the clean surface. The vibrational modes cause a $0.1 \%$ change in reflectivity, seen easily against "noise" which is about $5 \times$ lower than this. The scans were averaged over 3.5 minutes each. The best we have obtained is a reproducibility of 50 parts per million.

\section{Time Resolved Studies: Pump-probe}

Several studies have utilized the time structure of synchrotron radiation. In the infrared a significant advantage is obtained when working in the instantaneous rather than average power regime because the duty cycle advantage (roughly $\times 20$ ) pushes signals farther above detector noise. Fast photo-response measurements ${ }^{8}$ have been made on several high Tc materials to try to understand the dynamics of these systems at energies near the superconducting gap values. The fast response is dramatically shown in Fig. 8 in which we plot the signal as a function of time from a $\mathrm{YBaCuO}$ film and see the 7 filled if buckets of the NSLS VUV ring.

In addition we have recently collaborated with Ederer's group and established a fiber optic line to bring frequency doubled Nd-YAG laser pulses, synchronized (modelocked) to the NSLS VUV ring, to the infrared beamline. In these experiments the sample is excited by the $2 \mathrm{eV}$ laser pulse which precedes the infrared synchrotron pulse by a time between 0 and 10 nsecs. The synchrotron pulse then acts as a strobe, enabling a spectrum to be obtained of the system in its excited state 0 -10nsecs after the excitation proceeds.

\section{Summary}

Prior to this work all the instrumentation used with synchrotron radiation in the infrared was purchased commercially. We have developed the first instrumentation 
specifically for this source and the work continues. In addition to the experiments discussed above, the NSLS beamline has supported full programs measuring the far-ir transmission in superconductors, $\mathrm{C}_{60}$, proteins and in high pressure cells.

\section{Acknowledgements}

Work performed in collaboration with Carol Hirschmugl, Yves Chabal, Fritz Hoffmann, Dieter Moeller, Larry Carr and Peter Siddons and supported by the United States Department of Energy under contract no. DE-AC02-76CH00016. 


\section{References}

1. T. Nanba, Rev. Sci. Instrum. 60, 1680 (1989).

2. G. P. Williams, Nucl. Instr. and Methods A291, 8 (1990).

3. E. Schweitzer, J. Nagel, W. Braun, E. Lippert and A. M. Bradshaw, Nucl. Intr. \& Methods in Phys. Res. A239, 630 (1985).

4. M. Born and E. Wolf, Principles of Optics, (Pergamon Press, Oxford 1964).

5. K. D. Moller, D. P. Siddons, C. J. Hirschmugl, D. Scardino, P. Petrone, D. Carlson and G. P. Williams, Applied Optics, in press.

6. R. Madden and J. Strong, J. Opt. S c. Am. 44, 352 (1954).

7. C. J. Hirschmugl, G. P. Williams, F. M. Hoffmann and Y. J. Chabal, Physical Review Letters 65408 (1990).

8. G. L. Carr, M. Quijada, D. B. Tanner, C. J. Hirschmugl, G. P. Williams, S. Etemad, B. Dutta, F. DeRosa and T. Venkatesan, Applied Physics Letters 572725, (1990). 


\section{Figure Captions}

Fig. 1:(a) Schematic of a Michelson Interferometer in which the moving mirror moves a maximum distance $\mathrm{L} / 2$ giving a maximum path difference of $\mathrm{L}$. (b) Schematic of a lamellar grating in which one set of reflectors moves relative to a fixed set. The maximum distance scanned is $L / 2$ giving a maximum path difference of $L$.

Fig. 2:(a) Illustrating the condition for spatial coherence, which is that a source is said to be spatially coherent if the light passing through the apertures yields detectable interference fringes. (b) An alternative way of expressing the condition for spatial coherence, for which $2 \mathrm{D}=0.16 \lambda \mathrm{R} / \mathrm{s}$ or $\mathrm{s} . \alpha<<\lambda$.

Fig. 3. Schematic of a wavefront dividing interferometer for a spatially coherent synchrotron source which reflects from the two-component mirror. All rays are recombined at an image point. As the upper mirror is scanned, a path difference, $2 \mathrm{~d}$, is created between rays reflected from it and reference rays reflected from the lower mirror. Thus the instrument works like an interferometer without the need for an amplitude dividing beamsplitter.

Fig. 4. Interferogram obtained with an interferometer of the type shown in Fig. 3 for a polystyrene sample. The interferogram was recorded using 512 data points each averaged for $1 \mathrm{sec}$. A chopping frequency of $13 \mathrm{~Hz}$ was used and the detector was a liquid helium cooled bolometer. The detected interferogram was limited by filters and the detector to the region $50-800 \mathrm{~cm}^{-1}$.

Fig. 5. Fourier transform of Fig. 4 showing the spectrum in the region $50-800 \mathrm{~cm}^{-1}$. The overall shape is controlled primarily by the filters in the detector and the detector response. The absorption peaks are due to both the polystyrene sample and the filters.

Fig. 6. Photograph of the high resolution interferometer built by Dieter Moller for use 
at the NSLS infrared beamline. The inner set of mirrors moves, lengthening one path while shortening the other to produce a total path difference of 5 meters.

Fig. 7. Vibrational modes for a $c(2 \times 2)$ (half monolayer) coverage of $\mathrm{CO}$ on $\mathrm{Cu}(100)$ showing the isotopic shifts. The "frustrated rotation" mode at lower frequency has a Fano line-shape. The other mode is one in which the whole molecule vibrates perpendicular to the surface. What is plotted is the difference between the clean and $\mathrm{CO}$ covered surface. The peaks themselves represent a change in reflectivity of only $-0.1 \%$, the noise is at least $5 \times$ smaller than this. The data were taken in only 7 minutes.

Fig. 8. Signal from a high Tc superconductor used as a high speed bolometer detector, as a function of time. The 7 bunches circulating in the NSLS VUV ring are clearly visible. 


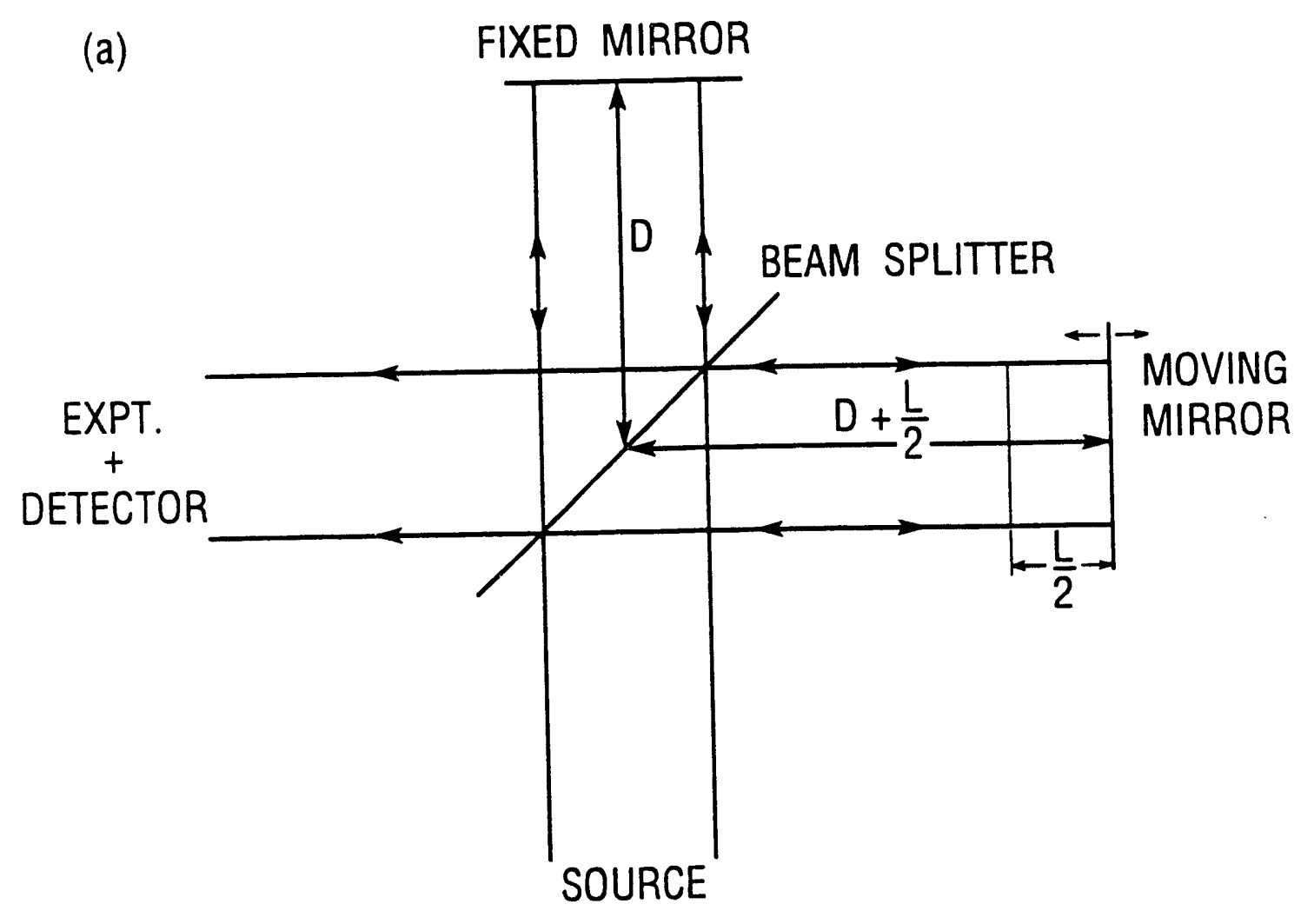

(b)

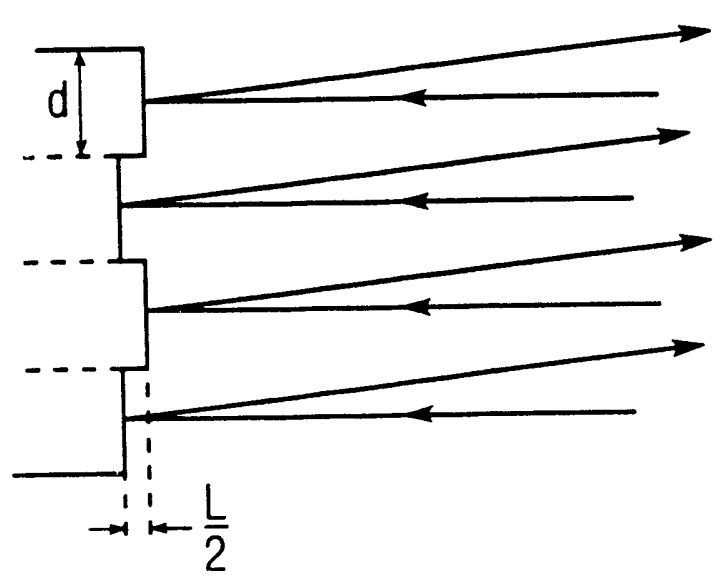




$$
\because
$$

(a)

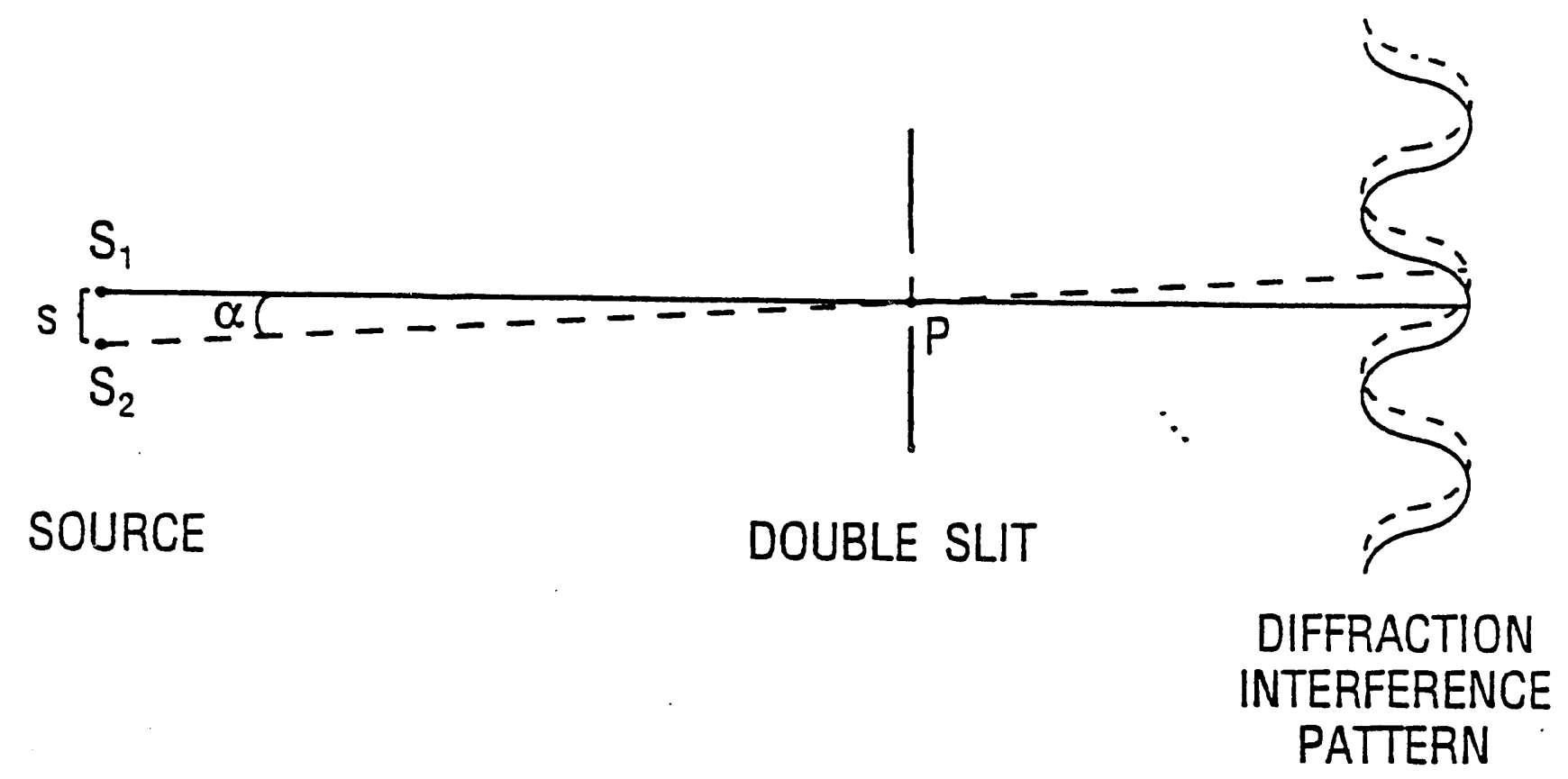

(b)

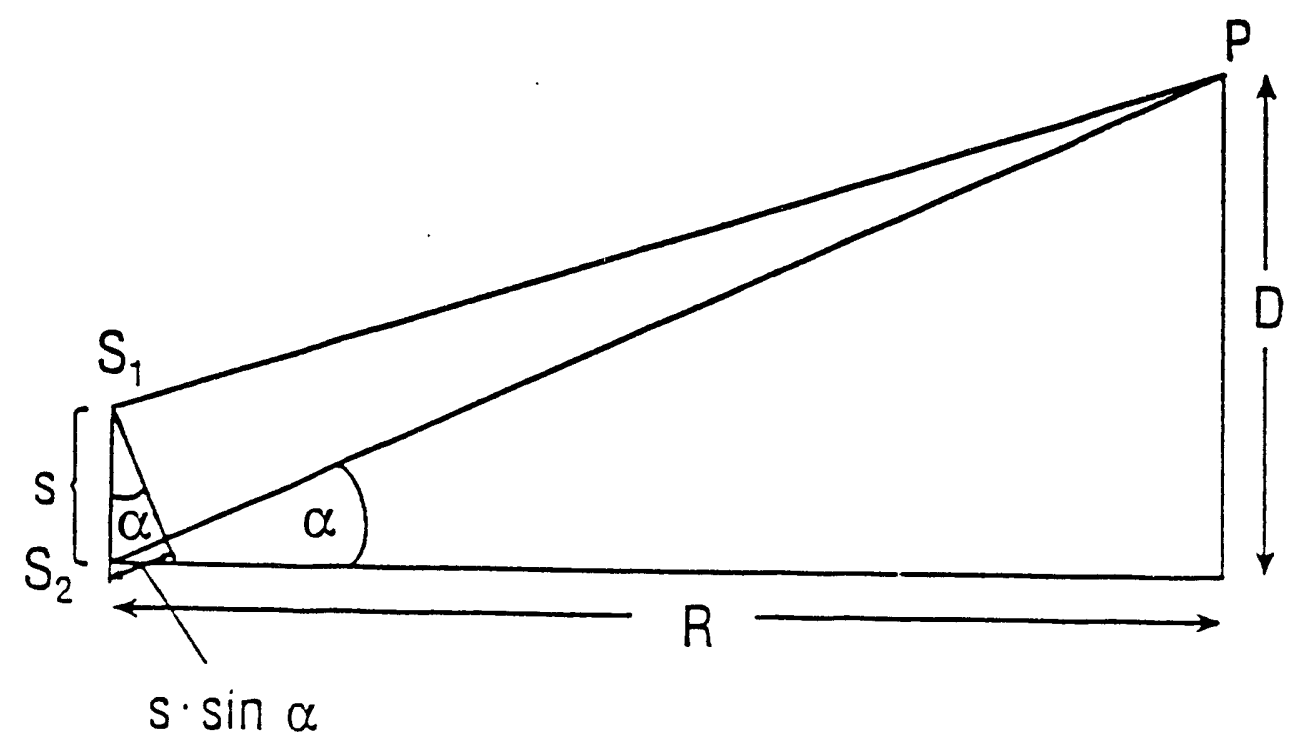




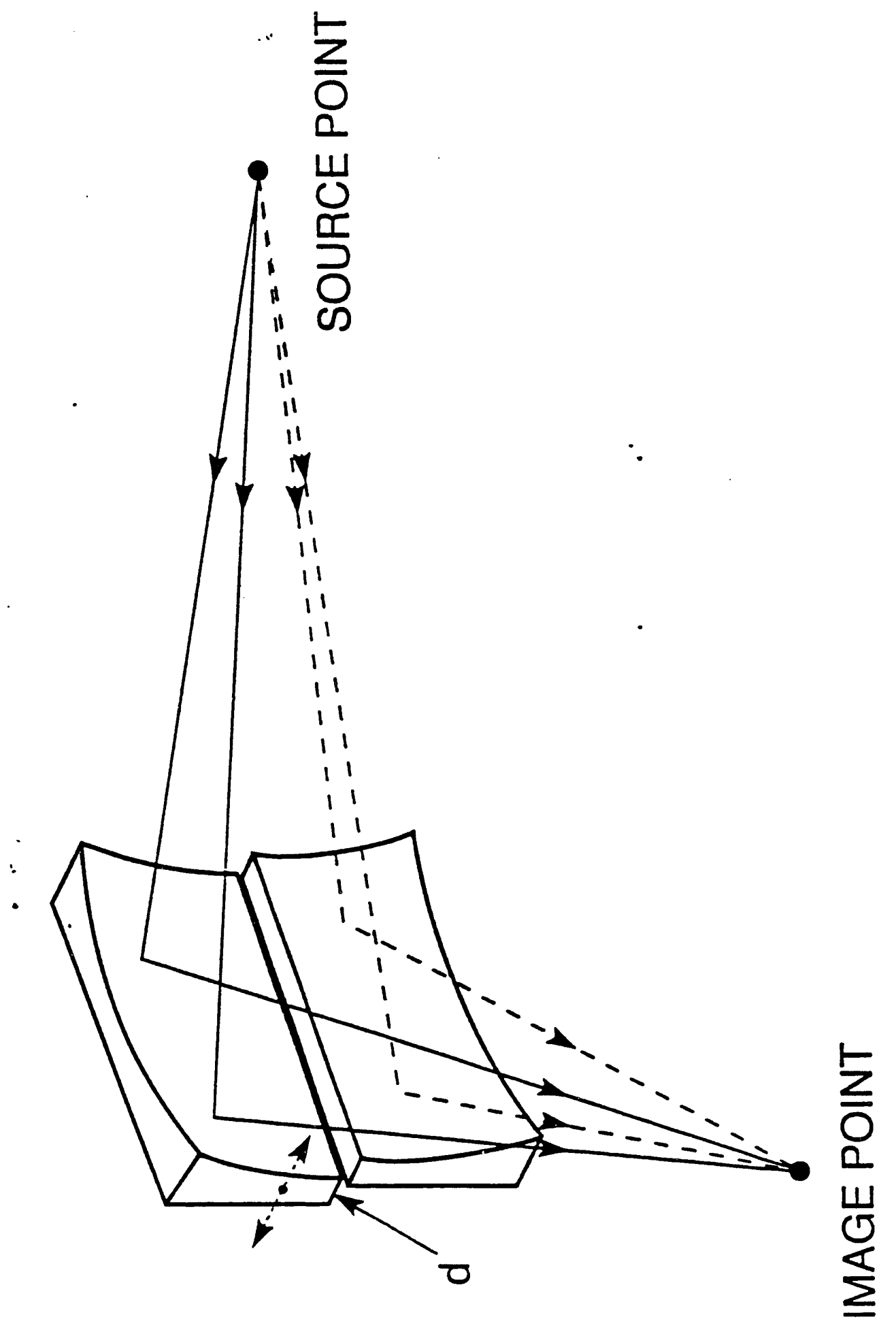




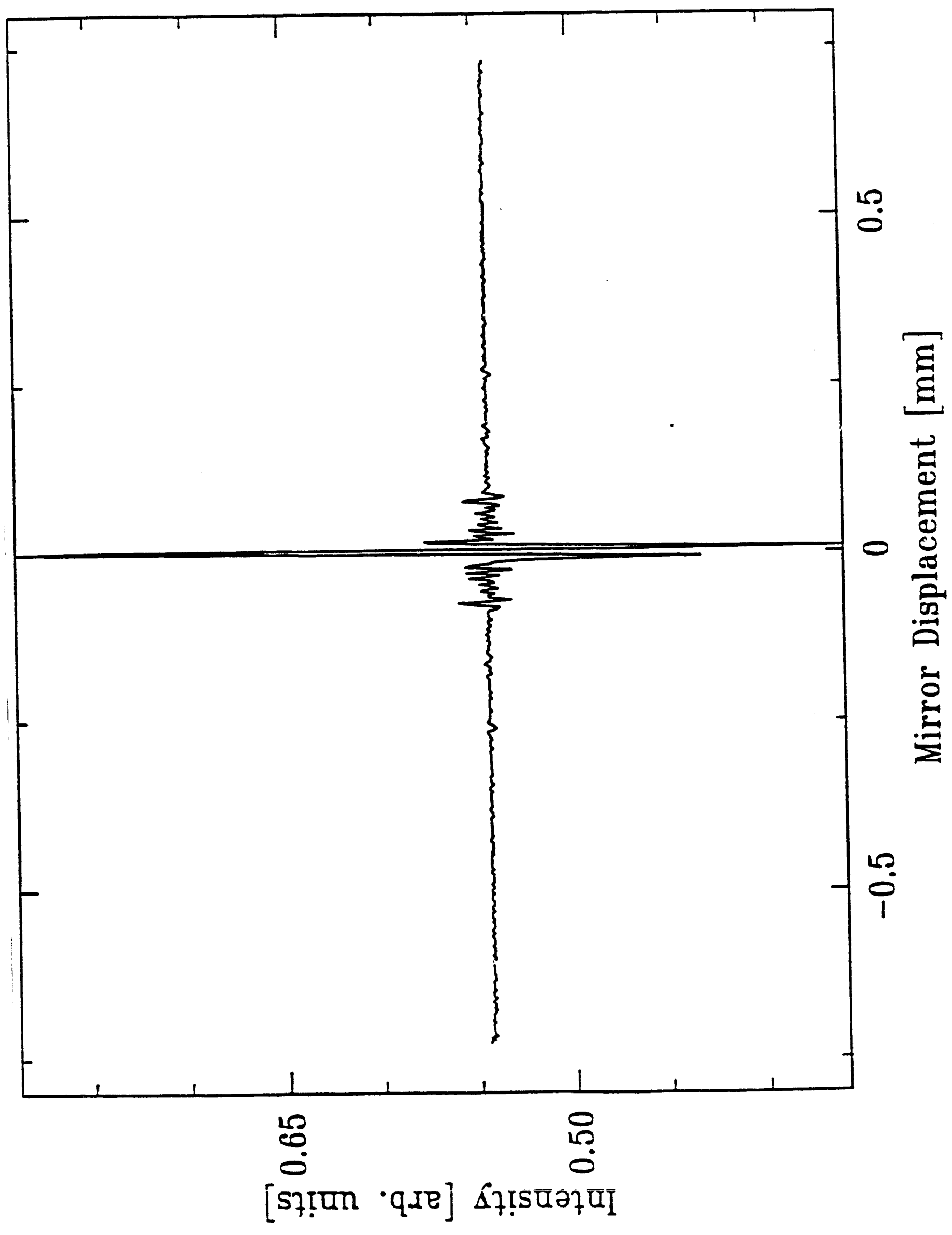




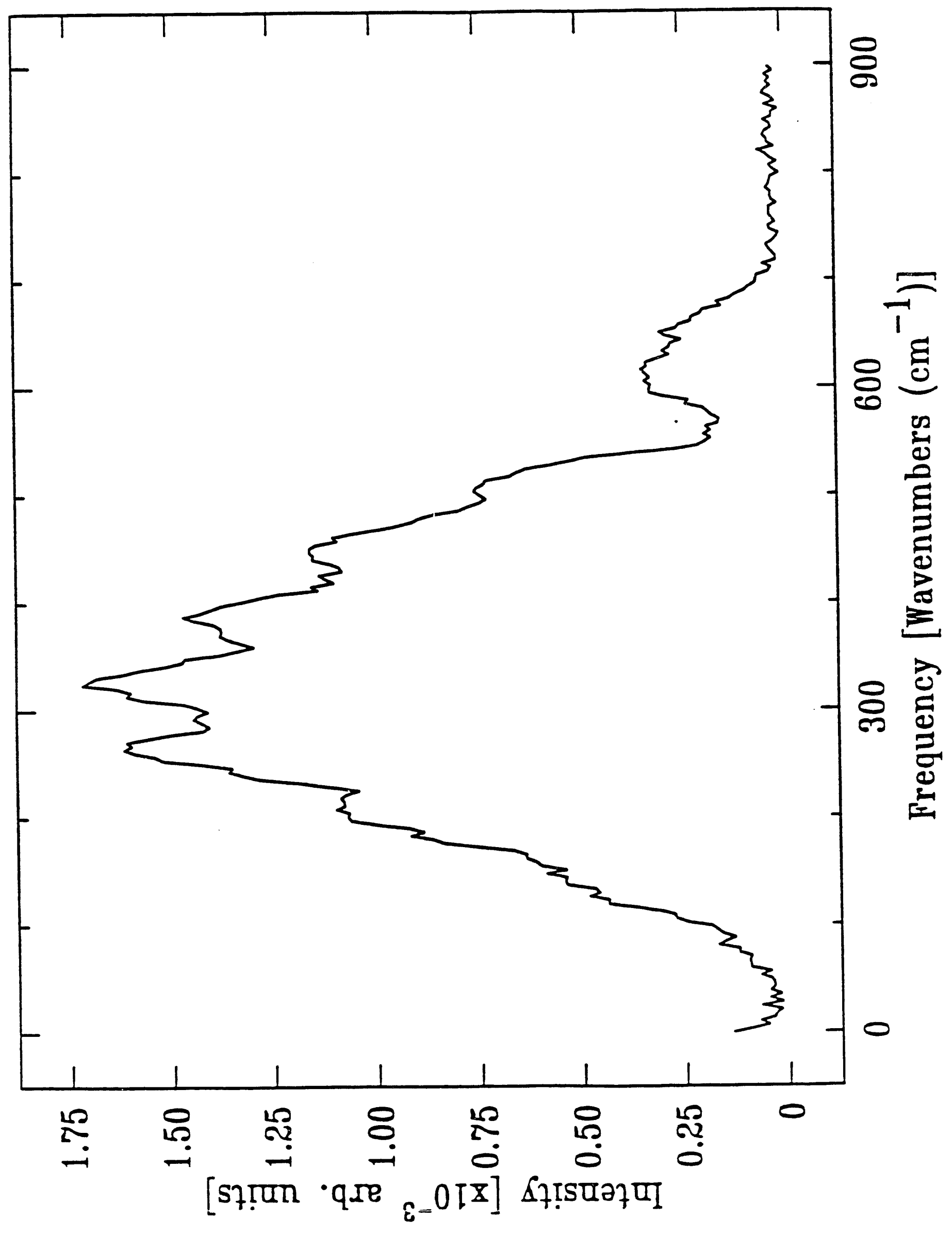




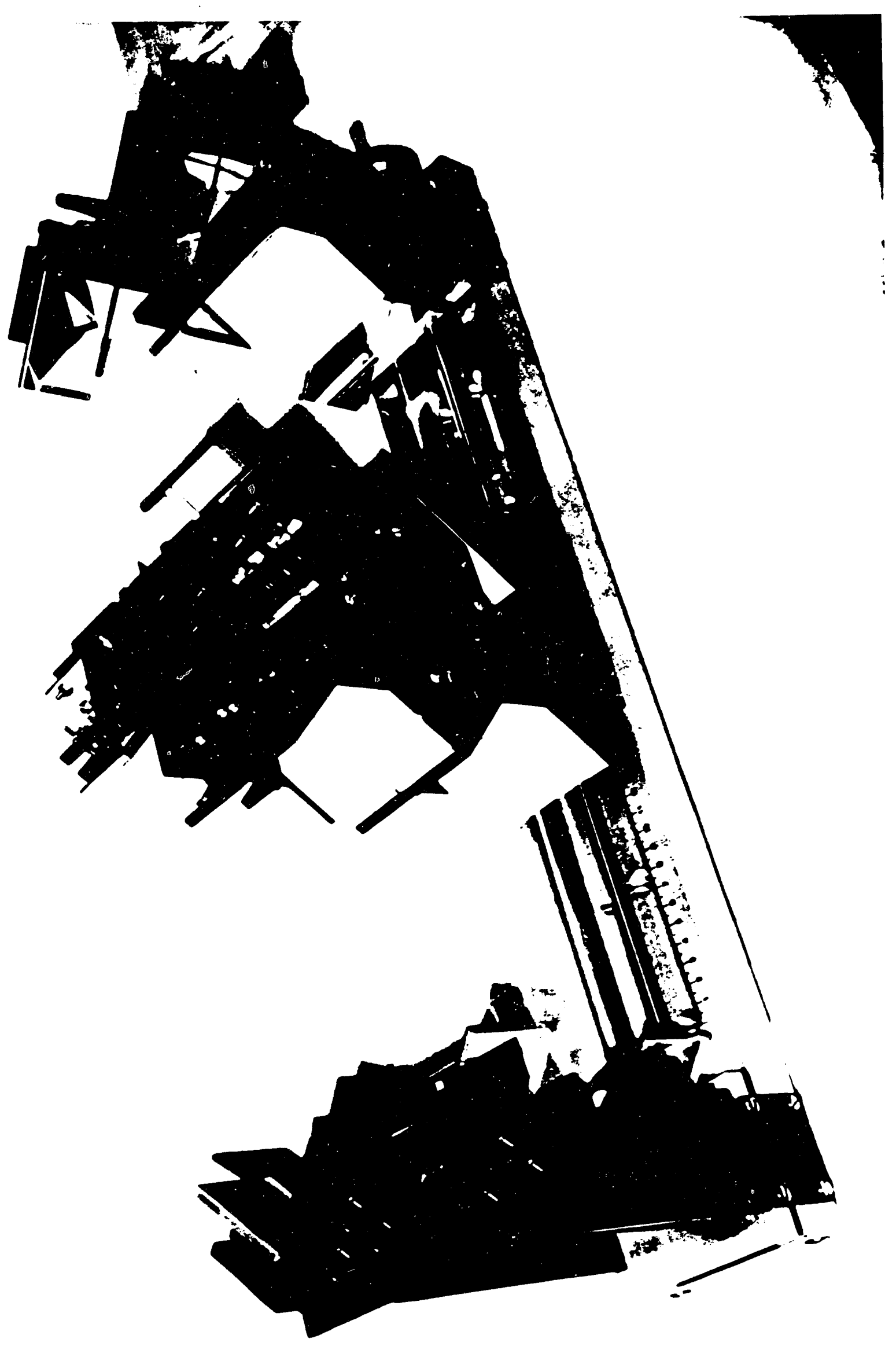




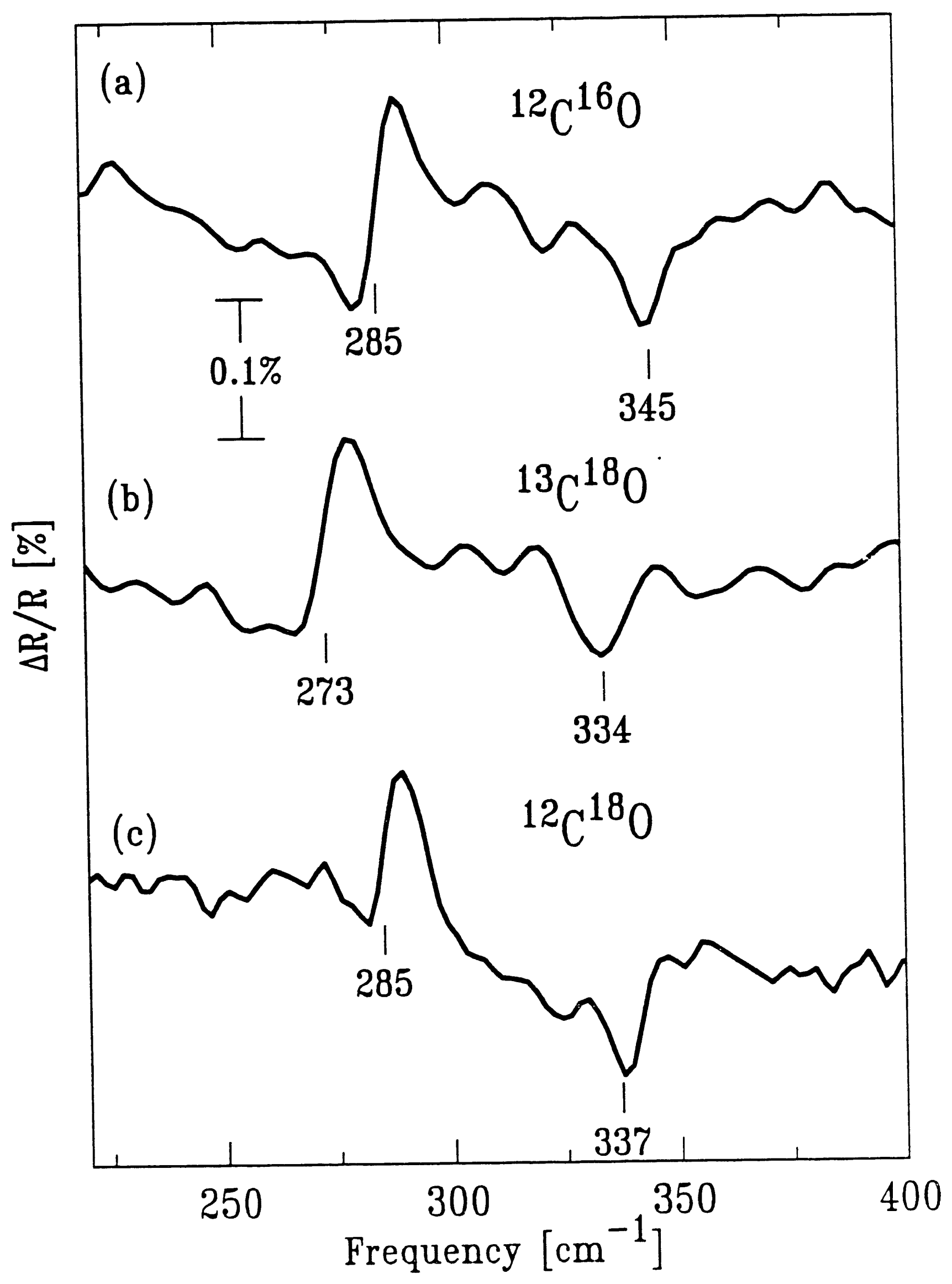




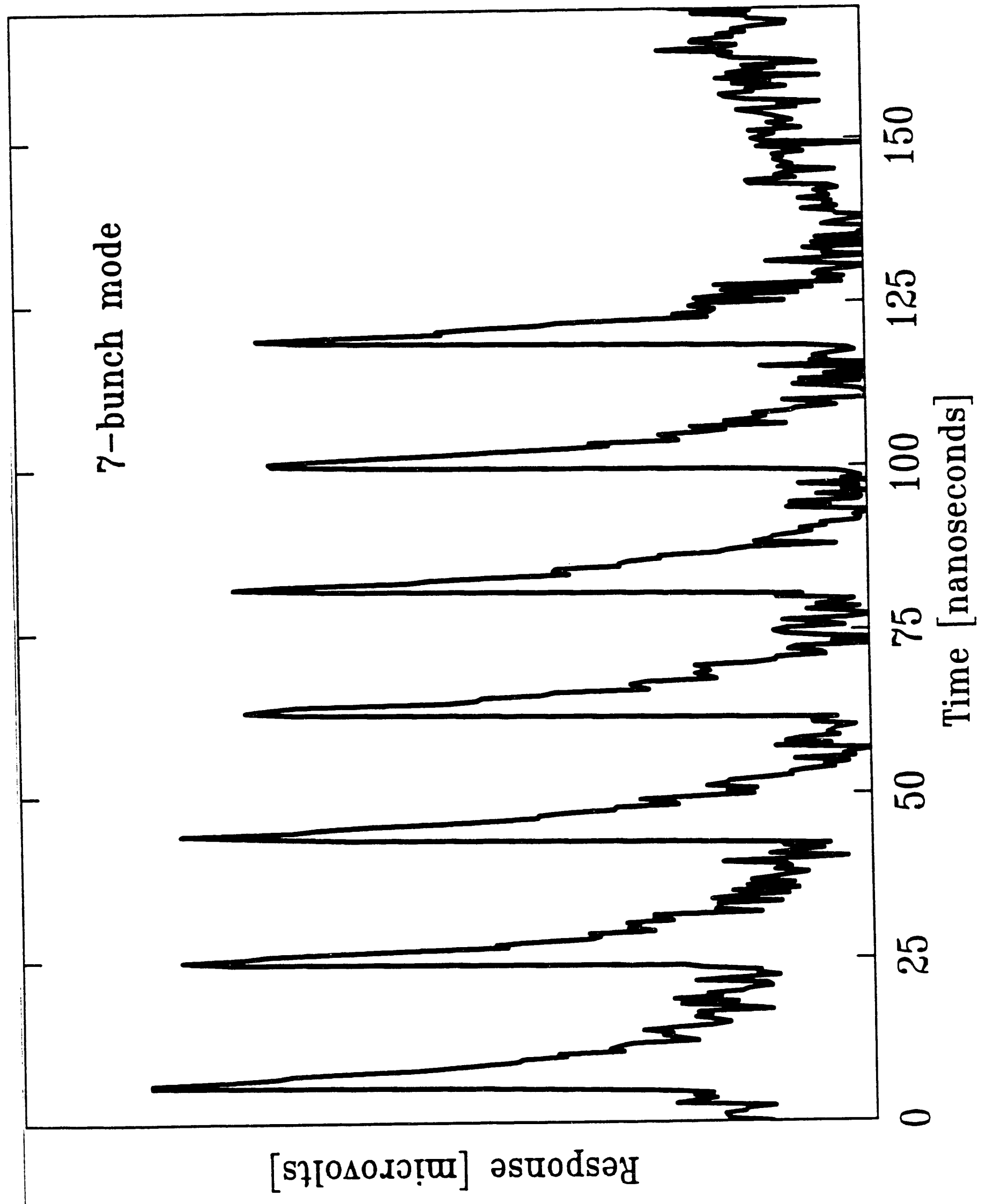



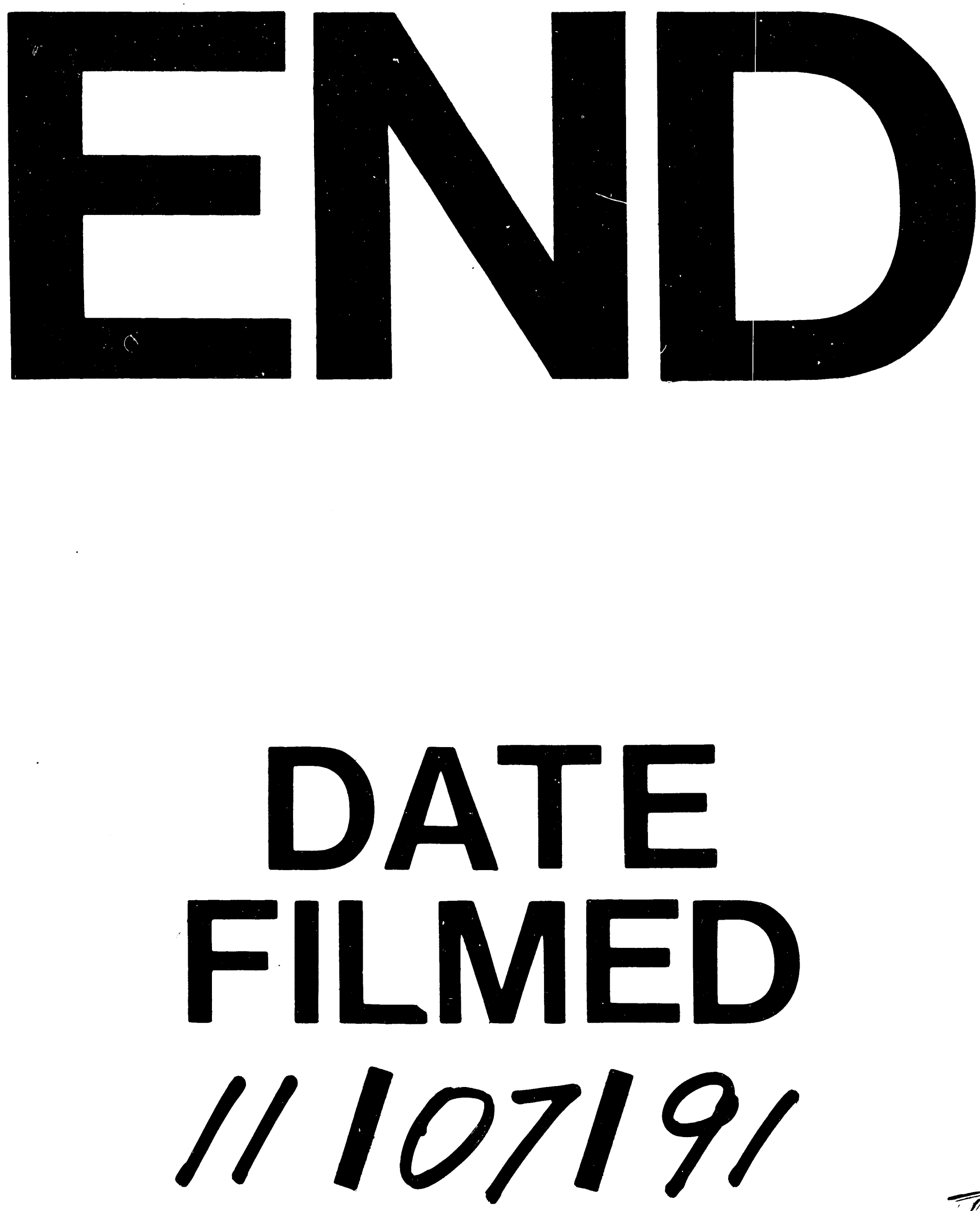

$\bar{T}$ 
